\title{
Tender Subcutaneous Nodule in a Prepubescent Boy
}

\author{
Maria M. Hornberger, MD; James J. Jones Jr, MD; Wendi E. Wohltmann, MD; Brittany L. Lenz, MD
}

\section{Eligible for 1 MOC SA Credit From the ABD}

This Dermatopathology Diagnosis in our print edition is eligible for 1 self-assessment credit for Maintenance of Certification from the American Board of Dermatology (ABD). After completing this activity, diplomates can visit the ABD website (http://www.abderm.org) to self-report the credits under the activity title "Cutis Dermatopathology Diagnosis." You may report the credit after each activity is completed or after accumulating multiple credits.

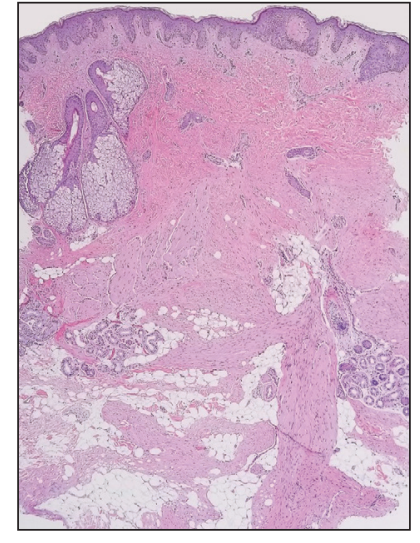

H\&E, original magnification $\times 40$.

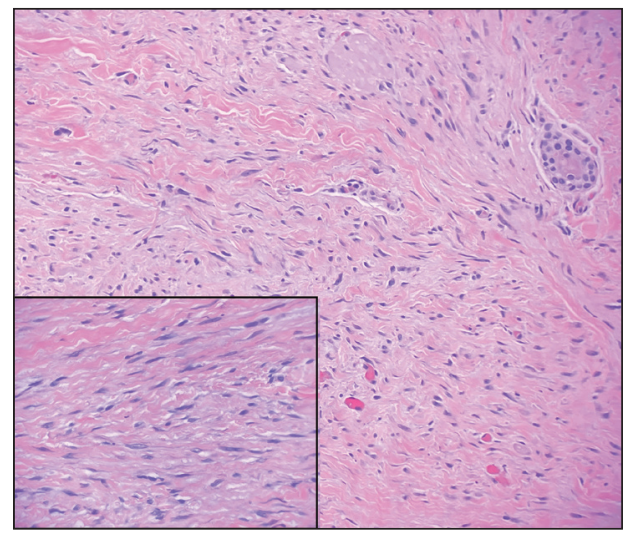

H\&E, original magnification $\times 200$ (inset, original magnification $\times 400$ ).
A 12-year-old boy with olive skin presented with a tender subcutaneous nodule on the back of 6 months' duration. He reported the lesion initially grew rapidly with increasing pain for approximately 3 months with subsequent stabilization in size and modest resolution of his symptoms. Physical examination revealed a solitary, 15-mm, ill-defined, indurated, tender, subcutaneous nodule with subtle overlying hyperpigmentation on the left side of the upper back. Hematoxylin and eosin staining of a 4-mm punch biopsy revealed a nonencapsulated mass of monomorphic eosinophilic spindle cells organized into fascicles arranged predominantly parallel to the skin surface. The mass extended from the mid reticular dermis to the upper subcutis, sparing adnexal structures.

\section{THE BEST DIAGNOSIS IS:}
a. atrophic dermatofibrosarcoma protuberans
b. dermatofibroma
c. dermatomyofibroma
d. piloleiomyoma
e. solitary cutaneous myofibroma

PLEASE TURN TO PAGE 358 FOR THE DIAGNOSIS

From the San Antonio Uniformed Services Health Education Consortium, Texas. Dr. Hornberger is from the Transitional Year Program, and Drs. Jones, Wohltmann, and Lenz are from the Department of Dermatology.

The authors report no conflict of interest.

The views presented do not represent the official views of the Department of Defense or its components.

Correspondence: Maria M. Hornberger, MD, 3551 Roger Brooke Dr, JBSA Ft Sam Houston, TX 78234 (maria.m.hornberger.mil@mail.mil). doi:10.12788/cutis.0405 


\section{THE DIAGNOSIS: Dermatomyofibroma}

$\square$ ermatomyofibroma is an uncommon, benign, cutaneous mesenchymal neoplasm composed of fibroblasts and myofibroblasts. ${ }^{1-3}$ This skin tumor was first described in 1991 by Hugel ${ }^{4}$ in the German literature as plaquelike fibromatosis. Pediatric dermatomyofibromas are exceedingly rare, with pediatric patients ranging in age from infants to teenagers. ${ }^{1}$

Clinically, dermatomyofibromas appear as long-standing, isolated, ill-demarcated, flesh-colored, slightly hyperpigmented or erythematous nodules or plaques that may be raised or indurated. ${ }^{1}$ Dermatomyofibromas may present with constant mild pain or pruritus, though in most cases the lesions are asymptomatic. ${ }^{1,3}$ The clinical presentation of dermatomyofibroma has a few distinct differences in children compared to adults. In adulthood, dermatomyofibroma has a strong female predominance and most commonly is located on the shoulder and adjacent upper body regions, including the axilla, neck, upper arm, and upper trunk..$^{1-3}$ In childhood, the majority of dermatomyofibromas occur in young boys and usually are located on the neck with other upper body regions occurring less frequently. ${ }^{1,2}$ A shared characteristic includes the tendency for dermatomyofibromas to have an initial period of enlargement followed by stabilization or slow growth. ${ }^{1}$ Reported pediatric lesions have ranged in size from 4 to $60 \mathrm{~mm}$ with an average size of $14.9 \mathrm{~mm}$ (median, $12 \mathrm{~mm}$ ). ${ }^{2}$

The diagnosis of dermatomyofibroma is based on histopathologic features in addition to clinical presentation. Histology from punch biopsy usually reveals a noninvasive dermal proliferation of bland, uniform, slender spindle cells oriented parallel to the overlying epidermis with increased and fragmented elastic fibers..$^{1,3}$ Infiltration into the mid or deep dermis is common. The adnexal structures usually are spared; the stroma contains collagen and increased small blood vessels; and there typically is no inflammatory infiltrate, except for occasional scattered mast cells. ${ }^{2}$ Cytologically, the monomorphic spindleshaped tumor cells have an ill-defined, pale, eosinophilic cytoplasm and nuclei that are elongated with tapered edges. $^{3}$ Dermatomyofibroma has a variable immunohistochemical profile, as it may stain focally positive for CD34 or smooth muscle actin, with occasional staining of factor XIIIa, desmin, calponin, or vimentin..$^{1-3}$ Normal to increased levels of often fragmented elastic fibers is a helpful clue in distinguishing dermatomyofibroma from dermatofibroma, hypertrophic scar, dermatofibrosarcoma protuberans, and pilar leiomyoma, in which elastic fibers typically are reduced. ${ }^{3}$ Differential diagnoses of mesenchymal tumors in children include desmoid fibromatosis, connective tissue nevus, myofibromatosis, and smooth muscle hamartoma. ${ }^{1}$
A punch biopsy with clinical observation and followup is recommended for the management of lesions in cosmetically sensitive areas or in very young children who may not tolerate surgery. In symptomatic or cosmetically unappealing cases of dermatomyofibroma, simple surgical excision remains a viable treatment option. Recurrence is uncommon, even if only partially excised, and no instances of metastasis have been reported. ${ }^{1-5}$

Dermatomyofibromas may be mistaken for several other entities both benign and malignant. For example, the benign dermatofibroma is the second most common fibrohistiocytic tumor of the skin and presents as a firm, nontender, minimally elevated to dome-shaped papule that usually measures less than or equal to $1 \mathrm{~cm}$ in diameter with or without overlying skin changes. ${ }^{5,6}$ It primarily is seen in adults with a slight female predominance and favors the lower extremities. ${ }^{5}$ Patients usually are asymptomatic but often report a history of local trauma at the lesion site. ${ }^{6}$ Histologically, dermatofibroma is characterized by a nodular dermal proliferation of spindleshaped fibrous cells and histiocytes in a storiform pattern (Figure 1). ${ }^{6}$ Epidermal induction with acanthosis overlying the tumor often is found with occasional basilar hyperpigmentation. ${ }^{5}$ Dermatofibroma also characteristically has trapped collagen ("collagen balls") seen at the periphery.,

Piloleiomyomas are benign smooth muscle tumors arising from arrector pili muscles that may be solitary

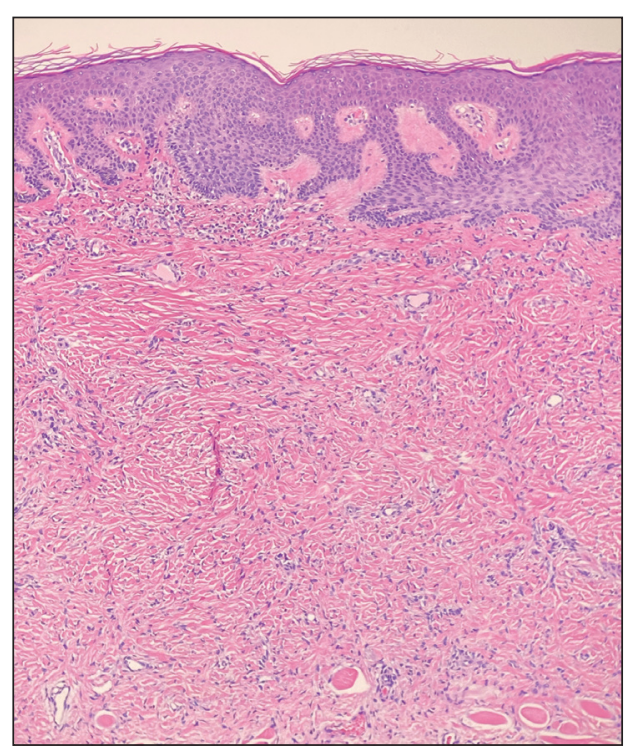

FIGURE 1. Dermatofibroma. Fibrohistiocytic proliferation in a storiform pattern with overlying epidermal induction and peripheral collagen trapping $(H \& E$, original magnification $\times 100)$. 
or multiple. ${ }^{5}$ Clinically, they typically present as firm, reddish-brown to flesh-colored papules or nodules that develop more commonly in adulthood. 5,7 Piloleiomyomas favor the extremities and trunk, particularly the shoulder, and can be associated with spontaneous or induced pain. Histologically, piloleiomyomas are well circumscribed and centered within the reticular dermis situated closely to hair follicles (Figure 2). ${ }^{5}$ They are composed of numerous interlacing fascicles or whorls of smooth muscle cells with abundant eosinophilic cytoplasm and blunt-ended, cigar-shaped nuclei. ${ }^{5,7}$

Solitary cutaneous myofibroma is a benign fibrous tumor found in adolescents and adults and is the counterpart to infantile myofibromatosis. ${ }^{8}$ Clinically, myofibromas typically present as painless, slow-growing, firm nodules with an occasional bluish hue. Histologically, solitary cutaneous myofibromas appear in a biphasic pattern, with hemangiopericytomatous components as well as spindle cells arranged in short bundles and fascicles resembling leiomyoma (Figure 3). The spindle cells also have abundant eosinophilic cytoplasm with short plump nuclei; the random, irregularly intersecting angles can be used to help differentiate myofibromas from smooth muscle lesions. ${ }^{8}$ Solitary cutaneous myofibroma is in the differential diagnosis for dermatomyofibroma because of their shared myofibroblastic nature. ${ }^{9}$

Dermatofibrosarcoma protuberans (DFSP) is an uncommon, locally invasive sarcoma with a high recurrence rate that favors young to middle-aged adults, with rare childhood onset reported. ${ }^{5,10,11}$ Clinically, DFSP typically presents as an asymptomatic, slow-growing, firm, flesh-colored, indurated plaque that develops into a violaceous to reddish-brown nodule. ${ }^{5}$ The atrophic variant of DFSP is characterized by a nonprotuberant lesion and can be especially difficult to distinguish from other entities

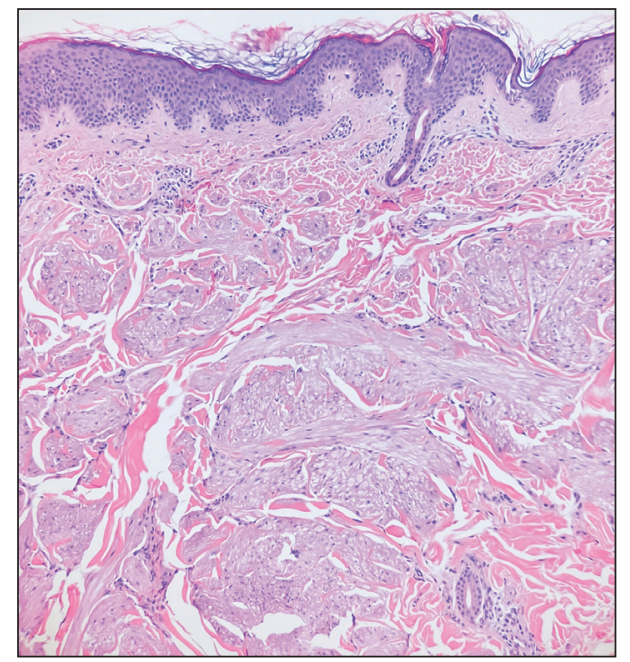

FIGURE 2. Piloleiomyoma. Proliferation of smooth muscle arranged in longitudinal fascicles with blunt, cigar-shaped nuclei and eosinophilic cytoplasm (H\&E, original magnification $\times 200)$. such as dermatomyofibroma. ${ }^{11}$ The majority of DFSP lesions occur on the trunk, particularly in the shoulder or pelvic region. ${ }^{5}$ Histologically, early plaque lesions are comprised of monomorphic spindle cells arranged in long fascicles (parallel to the skin surface), infiltrating adnexal structures, and subcutaneous adipocytes in a multilayered honeycomb pattern; the spindle cells of late nodular lesions are arranged in short fascicles in a matted or storiform pattern (Figure 4)..$^{5,10}$ Early stages of DFSP as well as variations in childhood-onset DFSP can easily be misdiagnosed and incompletely excised. ${ }^{5}$

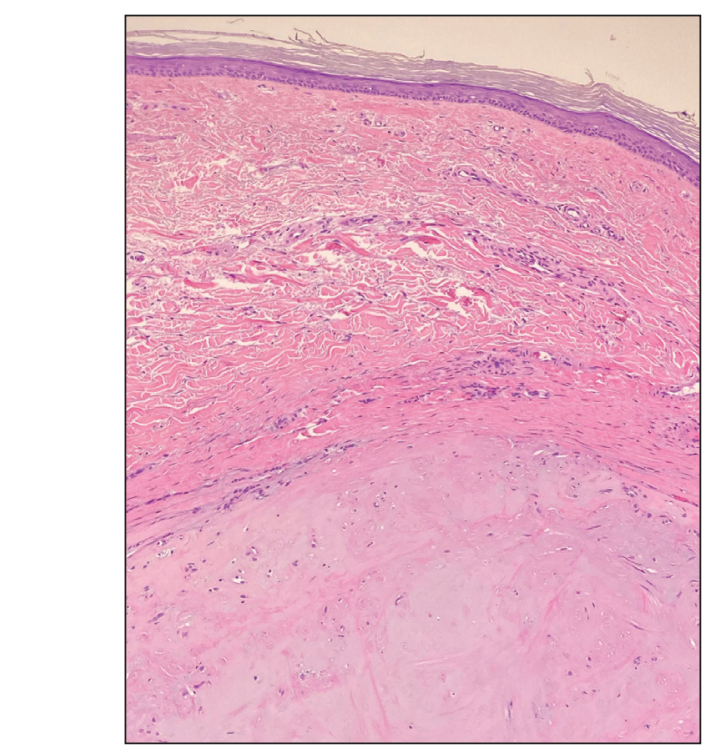

FIGURE 3. Solitary cutaneous myofibroma. Biphasic tumor nodule resembling myofibroblasts (fusiform cells with short plump nuclei and abundant eosinophilic cytoplasm) centrally and pericytes peripherally (H\&E, original magnification $\times 200$ ).

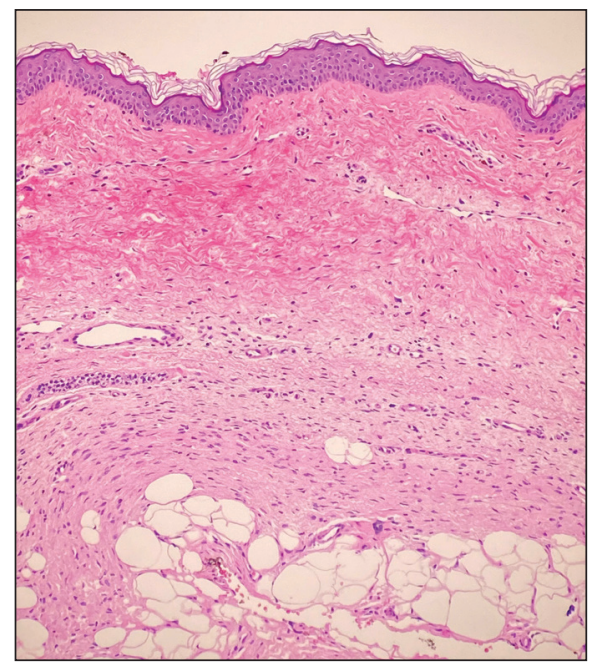

FIGURE 4. Atrophic dermatofibrosarcoma protuberans. Monomorphic spindle cells infiltrating adipocytes in a honeycomb pattern (H\&E, original magnification $\times 200$ ). 


\section{REFERENCES}

1. Ma JE, Wieland CN, Tollefson MM. Dermatomyofibromas arising in children: report of two new cases and review of the literature. Pediatr Dermatol. 2017;34:347-351.

2. Tardio JC, Azorin D, Hernandez-Nunez A, et al. Dermatomyofibromas presenting in pediatric patients: clinicopathologic characteristics and differential diagnosis. J Cutan Pathol. 2011;38:967-972.

3. Mentzel T, Kutzner H. Dermatomyofibroma: clinicopathologic and immunohistochemical analysis of 56 cases and reappraisal of a rare and distinct cutaneous neoplasm. Am J Dermatopathol. 2009;31:44-49.

4. Hugel H. Plaque-like dermal fibromatosis. Hautarzt. 1991;42:223-226.

5. Bolognia JL, Jorizzo J,, Schaffer JV, eds. Dermatology. WB Saunders Co; 2012.
6. Myers DJ, Fillman EP. Dermatofibroma. StatPearls [Internet]. StatPearls Publishing; 2020

7. Dilek N, Yuksel D, Sehitoglu I, et al. Cutaneous leiomyoma in a child: a case report. Oncol Lett. 2013;5:1163-1164.

8. Roh HS, Paek JO, Yu HJ, et al. Solitary cutaneous myofibroma on the sole: an unusual localization. Ann Dermatol. 2012;24:220-222.

9. Weedon D, Strutton G, Rubin AI, et al. Weedon's Skin Pathology. Churchill Livingstone/Elsevier; 2010

10. Mendenhall WM, Zlotecki RA, Scarborough MT. Dermatofibrosarcoma protuberans. Cancer. 2004;101:2503-2508.

11. Akay BN, Unlu E, Erdem C, et al. Dermatoscopic findings of atrophic dermatofibrosarcoma protuberans. Dermatol Pract Concept. 2015;5:71-73. 